\title{
Limited Binding Capacity Sites for L-Triiodothyronine in Rat Liver Nuclei
}

\author{
NUCLEAR-CYTOPLASMIC INTERRELATION, BINDING \\ CONSTANTS, AND CROSS-REACTIVITY WITH L-THYROXINE
}

\author{
Jack H. Oppenheimer, Harold L. Schwartz, Diona Koerner, and \\ MARTIN I. SURKS \\ From the Endocrine Research Laboratory, Division of Endocrinology, \\ Department of Medicine, Montefiore Hospital and Medical Center and the \\ Albert Einstein College of Medicine, Yeshiva University, \\ Bronx, New York 10467
}

\begin{abstract}
A в S T R A C T Further studies have been performed to define the kinetic characteristics of nuclear triiodothyronine $\left(\mathrm{T}_{3}\right)$ binding sites in rat liver (J. Clin. Endocrinol. Metab. 1972. $35: 330)$. Sequential determination of labeled $\mathrm{T}_{3}$ associated with nuclei and cytoplasm over a 4-h period allowed analysis of the relationship of $\mathrm{T}_{3}$ in nuclear and cytoplasmic compartments. A rapid interchange of hormone between nuclei and cytoplasm was demonstrated, and in vitro incubation experiments with nuclei yielded no evidence favoring metabolic transformation of $T_{3}$ by the nuclei. In vivo displacement experiments were performed by subcellular fractionation of liver $\frac{1}{2} \mathrm{~h}$ after injection of $\left[{ }^{125} \mathrm{I}\right] \mathrm{T}_{3}$ with increasing quantities of unlabeled $T_{3}$. The nuclear binding capacity for $\mathrm{T}_{3}$ could be defined $(0.52 \mathrm{ng} / \mathrm{mg} \mathrm{DNA})$. Analysis of these experiments also allowed an estimation of the association constant of nuclear sites for $\mathrm{T}_{3}(4.7 \times$ $\left.10^{11} \mathrm{M}^{-1}\right)$. The affinity of these sites for $\mathrm{T}_{3}$ was estimated to be $20-40$ fold greater than for thyroxine $\left(T_{4}\right)$. Chromatographic analysis of the nuclear radioactivity after injection of labeled $T_{4}$ indicated that the binding of $T_{4}$ by the nucleus could not be attributed to in vivo conversion of $T_{4}$ to $T_{3}$ but reflected intrinsic cross-reactivity of the two iodothyronines at the nuclear binding sites.
\end{abstract}

Dr. Surks is the recipient of a National Institutes of Health Research Career Development Award, KO 4AM 19502-01 A1.

Received for publication 19 March 1973 and in revised form 15 June 1973.

\section{INTRODUCTION}

We have previously demonstrated limited capacity nuclear binding sites for L-triiodothyronine $\left(\mathrm{T}_{3}\right)^{1}$ in rat liver and kidney nuclei (1). Our findings were based on in vivo displacement studies and suggested a high degree of specificity for $T_{3}$, since thyroxine $\left(T_{4}\right)$ appeared to be much less avidly bound to nuclei and the degree of displacement of radioactive $T_{4}$ by nonradioactive $T_{4}$ was far less pronounced than in the case of $\mathrm{T}_{3}$. Since Tata and Widnell (2) had previously shown that pulse injection of $T_{3}$ into thyroidectomized rats stimulates nuclear RNA metabolism before biochemical changes in other subcellular fractions, the finding of limited capacity nuclear sites suggested to us the possibility that such sites might represent hormonal "receptors" responsible for the initiation of the hormonal message. Accordingly, we initiated a series of studies designed to explore the nature and significance of these sites in greater detail. In the present communication, we have addressed ourselves to the following quantitative and kinetic problems: (a) the compartmental relationship between $T_{3}$ in nuclei and $T_{3}$ in cytoplasm of liver cells; $(b)$ measurement of the nuclear binding capacity of $T_{3}$ per gram of liver; and $(c)$ the extent of cross-reactivity of these sites between $T_{3}$ and $T_{4}$.

\section{METHODS}

The methods for measuring nuclear binding have been briefly reported in our previous communication (1). As

${ }^{1}$ Abbreviations used in this paper: $\mathrm{L} / \mathrm{P}$, liver to plasma ratio; $100 \mathrm{~N} / \mathrm{L}$, percent of total liver radioactivity associated with the nuclear pellet; $T_{3}, \mathrm{~L}$-triiodothyronine; $T_{4}$, thyroxine. 
indicated above, this technique depends upon displacement of $\left[{ }^{125} \mathrm{I}\right] \mathrm{T}_{8}$ by nonradioactive $\mathrm{T}_{3}$ in vivo. Male SpragueDawley rats weighing between 150 and $250 \mathrm{~g}$ were obtained from Carworth Div., Becton, Dickinson \& Co., New City, N. Y., and were maintained on a Wayne Lab Rat diet (1 $\mu \mathrm{g} \mathrm{I/g)} \mathrm{(Wayne} \mathrm{Kerr} \mathrm{Lab} \mathrm{Ltd.,} \mathrm{Chessington,} \mathrm{Surrey,}$ England). Animals were used within 2 wk of shipment. Rats were injected through the tail vein with doses of isotopic and nonisotopic iodothyronines in a $1 \%$ albumin solution. $\left[{ }^{125} \mathrm{I}\right] \mathrm{T}_{3}$ (sp act approximately $60-80 \mu \mathrm{Ci} / \mu \mathrm{g}$ ) and $\left.{ }^{125} \mathrm{I}\right] \mathrm{T}_{4}$ (sp act approximately $60-80 \mu \mathrm{Ci} / \mu \mathrm{g}$ ) were obtained from Abbott Laboratories (North Chicago, Ill.). Nonradioactive $T_{4}$ and $T_{3}$ were obtained from Mann Research Labs, Inc., New York. Unless otherwise indicated, animals were killed $30 \mathrm{~min}$ after injection by exsanguination under light ether. Blood was obtained from the abdominal aorta, after which the liver was removed, blotted, and processed at $0^{\circ} \mathrm{C}$. Approximately $4 \mathrm{~g}$ of liver was weighed and homogenized in 3.0 vol of $0.32 \mathrm{M}$ sucrose containing $2 \mathrm{mM} \mathrm{MgCl}_{2}$ in a mechanically driven Teflon pestle. The homogenate was brought to $10 \mathrm{vol}$ of $0.32 \mathrm{M}$ sucrose and centrifuged at $700 \mathrm{~g}$ for $10 \mathrm{~min}$. The $700 \mathrm{~g}$ pellet was resuspended in $2.4 \mathrm{M}$ sucrose and subjected to an additional centrifugation at $28,000 \mathrm{~g}$ for $45 \mathrm{~min}$ in a Spinco 40 rotor (Beckman Instruments, Inc., Spinco Div., Palo Alto, Calif.) (3). The purity of the nuclear preparations was checked by electron and phase microscopy. Radioactive measurements were made in a Packard automatic well scintillation counter with standard techniques with a statistical counting error of less than 5\% (Packard Instrument Co., Inc., Downer's Grove, Ill.). In the case of $\mathrm{T}_{3}$, chromatographic studies indicated that in the time interval studied, over $95 \%$ of the radioactivity in both the total liver homogenate and the purified nuclear pellet was in the form of $T_{3}$. After injection of $T_{4}$ the nuclei selectively bound the small quantity of radioactive $T_{3}$ derived both from the in vivo $T_{4}$ to $T_{3}$ conversion (4) and the trace contamination (up to $2 \%$ of total radioactivity) in the doses of radioactive $T_{4}$ used (5). The problem created by the selective retention of $T_{3}$ by nuclei will be discussed in detail in the Results section. Ethanolic extract of liver homogenate and nuclear pellets were subjected to paper chromatography in t-amyl alcohol: $2 \mathrm{~N}$ ammonia: hexane (6). Plasma samples were precipitated with trichloracetic acid to remove radioiodide. DNA determinations on total liver homogenate and nuclear pellets were performed according to the method of Burton (7). Plasma $T_{3}$ in a group of normal rats was measured by radioimmunoassay (8). In order t) assess the possibility that nuclei can metabolize $T_{3}$, liver nuclei were incubated for $1 \mathrm{~h}$ at $37^{\circ} \mathrm{C}$ with tracer $\left.{ }^{125} \mathrm{I}\right] \mathrm{T}_{3}$ in a Tris buffer ( $\mathrm{pH} 7.4)$ containing $1 \mathrm{mM} \mathrm{MgCl}_{2}, 1^{-3}$ $\mathrm{M}$ ATP, and a NADPH-generating system of $10^{-3} \mathrm{M}$ $\mathrm{NADH}, 0.1 \mathrm{M}$ glucose-6-phosphate, and glucose-6-phosphate dehydrogenase. Calculations used in the analysis will be discussed in conjunction with the results of individual experiments.

\section{RESULTS}

Compartmental relationship between $T_{3}$ in the nucleus and $T_{3}$ in the cytoplasm. Previous studies have indicated that $T_{3}$ is bound to nuclear constituents by noncovalent linkage, since the hormone can be easily extracted with ethanol. It was not clear, however, whether in the cell nuclear $T_{3}$ is readily exchangeable with $T_{8}$ in the cytoplasm ( $=$ extranuclear cellular $T_{8}$ ) under in vivo conditions. In an effort to answer this question, we analyzed the concentration of $\left[{ }^{125} \mathrm{I}\right] \mathrm{T}_{3}$ in the nucleus and cytoplasm (= homogenate radioactivity - nuclear radioactivity) in livers of animals killed $5 \mathrm{~min}, 15 \mathrm{~min}$, $30 \mathrm{~min}, 1.5 \mathrm{~h}, 2.5 \mathrm{~h}$, and $4 \mathrm{~h}$ after the injection of tracer hormone. ${ }^{2}$ Four animals were used at each time point. Paper chromatography of ethanolic extracts of nuclear pellets and total liver homogenates indicated that over $95 \%$ of the radioactivity was in the form of $\mathrm{T}_{\mathrm{s}}$. The dose of $\mathrm{T}_{3}$ injected ( $3.85 \mathrm{ng} \mathrm{T}_{\mathrm{s}} / 100 \mathrm{~g}$ body wt) was determined to be in the true tracer range by establishing that smaller quantities of hormone injected produced identical distribution of radioactivity among plasma, liver, and nuclei. The largest increments of $T_{s}$ in plasma due to injected hormone were achieved in animals killed $5 \mathrm{~min}$ after the injection and averaged $0.016 \mathrm{ng} / \mathrm{ml}$, a value only $2 \%$ of the estimated normal $\mathrm{T}_{\mathrm{s}}$ plasma concentration. In other experiments we have shown that when the plasma $\mathrm{T}_{3}$ concentration is increased by less than $20 \%$ of its normal concentration, no alterations in the parti-

\begin{abstract}
${ }^{2}$ In this and following analyses we have assumed that the partition of $T_{3}$ between specific nuclear sites and the remainder of the cell as determined by differential centrifugation provides an accurate representation of the relationship that exists in the intact cells. This assumption appears reasonable since at $0^{\circ} \mathrm{C}$ there is extremely poor exchange of $\mathrm{T}_{3}$ between specific nuclear sites and the cytoplasm in vitro. Thus, nuclear binding of tracer $\mathrm{T}_{3}$ added to the liver homogenate at $0^{\circ} \mathrm{C}$ is much weaker than binding observed after in vivo injection of isotope (1). Conversely, we have added as much as $200 \mu \mathrm{g} \mathrm{T}_{3} / \mathrm{g}$ liver to the final homogenate without displacing in vivo injected tracer $T_{3}$. Moreover, when the final nuclear pellet is recentrifuged through sucrose, less than $2 \%$ of the radioactivity is lost. Finally, an experiment was carried out to determine whether dilution of the liver homogenate in increasing volumes of sucrose would promote the dissociation of $\mathrm{T}_{3}$. The liver homogenate was brought up to 5,10 , and 20 vol of $0.32 \mathrm{M}$ sucrose and centrifuged at $700 \mathrm{~g}$ for $10 \mathrm{~min}$. The pellet was resuspended and centrifuged at $28,000 \mathrm{~g}$ in heavy sucrose as described above. No consistent effect of dilution was noted $(1: 5,0.32 \%$ dose in the nuclear fraction per gram liver;
\end{abstract} $1: 10,0.34 \% ; 1: 20,0.32 \%$ ).

The possibility that nuclear $\mathrm{T}_{3}$ is instantaneously lost when the homogenate is placed in hypertonic sucrose should be considered. Although losses due to this mechanism cannot be easily estimated experimentally, theoretical considerations can serve to set an upper limit. Assume that $20 \%$ of hepatocellular binding is due to "specific" nuclear sites. If these are saturated, the liver: plasma ratio is reduced from about 10 to 8 , barely detectable given biological variations (1). If, on the other hand, we assume that $40 \%$ of liver binding was due to specific nuclear sites, then saturation would reduce the $\mathrm{L} / \mathrm{P}$ ratio from 10 to 6 , which should be easily detected. In the absence of an observable reduction in the $\mathrm{L} / \mathrm{P}$ ratio with saturating doses, one must consider that any possible losses due to "osmotic shock" are less than $50 \%$ of the amount originally bound to the limited-capacity nuclear sites. 


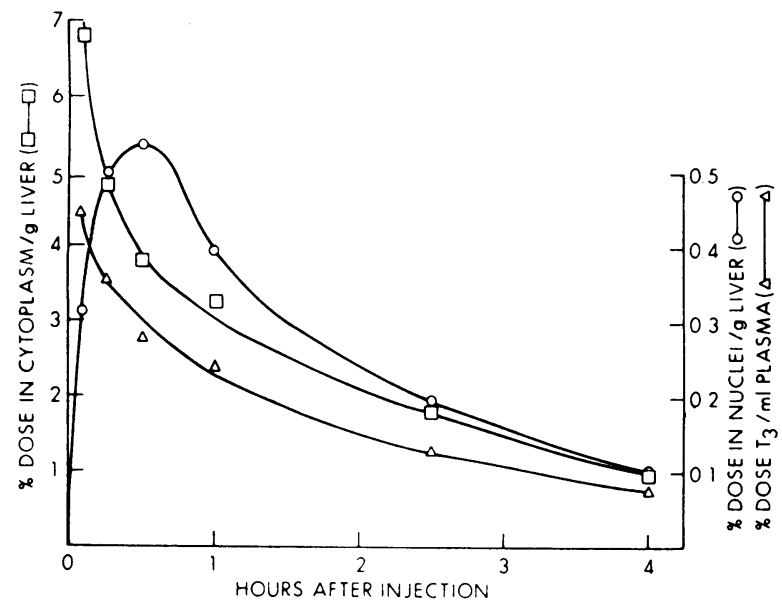

Figure 1 Time-course of concentration of radioactive $T_{3}$ in plasma, cytoplasm, and nuclei after intravenous injection of a single dose of tracer $\mathrm{T}_{3}$.

tion of hormone among nuclei, cytoplasm, and plasma are detectable.

The results of sequential analyses of radioactive $T_{3}$ distribution are illustrated in Fig. 1. A progressive fall in the concentration of radioactive $\mathrm{T}_{3}$ in plasma and cytoplasm was noted from the earliest point of observation $(5 \mathrm{~min})$. The concentration of nuclear $\left[{ }^{125} \mathrm{I}\right] \mathrm{T}_{3}$ rose to a peak at $30 \mathrm{~min}$ and thereafter appeared to approach a fractional decay rate similar to that of cytoplasm and plasma. The ratio between $\mathrm{T}_{3}$ in plasma and cytoplasm was constant throughout the experiment, a

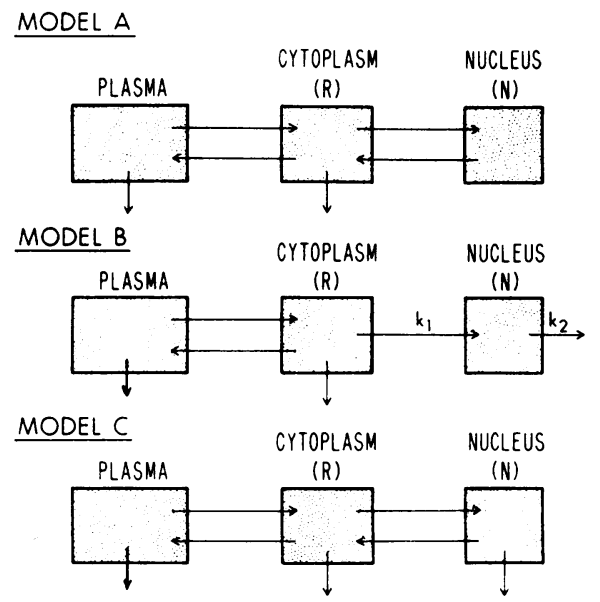

Figure 2 Three model systems describing the possible relationships between cytoplasm and nuclei. On the basis of data contained in Fig. $1, k_{1}$ and $k_{2}$ were evaluated for Model B. It was determined that the calculated value of $k_{2}$ led to an estimate of irreversible clearance rate of $T_{s}$ from the nuclei several times larger than entire metabolic clearance rate of the animal. Since Model B is not possible, there must be a return of $T_{3}$ from nuclei to cytoplasm as indicated in Model A or Model C. See text for details.
TABLE I

Nuclear Binding of $T_{3}$ in Euthyroid Animals

\begin{tabular}{|c|c|c|c|c|c|}
\hline 「Exp. & $\begin{array}{c}\text { Dose } \\
T_{\mathbf{8}} \\
\text { injected }\end{array}$ & $\begin{array}{c}\text { Hepatic } \\
T_{3} \\
\text { concn. }\end{array}$ & $\left(\frac{100 N}{L}\right)$ & $\left(\frac{100 N}{L}\right)$ & $\begin{array}{c}\mathrm{T}_{3} \text { bound } \\
\text { to } \\
\text { specific } \\
\text { nuclear } \\
\text { sites }\end{array}$ \\
\hline & $\begin{array}{c}n g / 100 \mathrm{~g} \\
\text { body wt }\end{array}$ & $n g / g$ liver & & & $n g / g$ liver \\
\hline \multirow[t]{8}{*}{11} & 65 & 11.2 & 8.56 & 7.53 & 0.96 \\
\hline & 120 & 16.2 & 6.64 & 5.61 & 0.94 \\
\hline & 176 & 17.5 & 6.72 & 5.69 & 1.00 \\
\hline & 232 & 21.2 & 5.32 & 4.29 & 0.91 \\
\hline & 344 & 33.6 & 3.99 & 2.96 & 0.99 \\
\hline & 1,961 & 150.1 & 1.79 & 0.76 & 1.14 \\
\hline & 10,049 & 825.0 & 1.03 & - & $\ldots$ \\
\hline & & & & & av. 0.99 \\
\hline \multirow[t]{7}{*}{16} & 79 & 16.0 & 6.85 & 5.85 & 0.94 \\
\hline & 148 & 18.9 & 6.99 & 5.99 & 1.1 .3 \\
\hline & 288 & 32.4 & 5.42 & 4.42 & 1.4 .3 \\
\hline & 2,100 & 180.0 & 1.76 & 0.76 & 1.37 \\
\hline & 9,767 & 667.0 & 1.02) & - & $\ldots$ \\
\hline & 20,919 & $1,844.0$ & $0.98)^{1.00}$ & - & - \\
\hline & & & & & av. 1.22 \\
\hline \multirow[t]{7}{*}{$25 \mathrm{~A}$} & 5 & 9.4 & 12.54 & 10.68 & 1.01 \\
\hline & 230 & 24.7 & 5.89 & 4.03 & 1.00 \\
\hline & 575 & 41.0 & 4.73 & 2.87 & 1.18 \\
\hline & 2,047 & 149.0 & 2.82 & 0.96 & 1.4 .3 \\
\hline & 9,798 & 614.0 & $1.96)$ & - & $\ldots$ \\
\hline & 20,092 & $1,263.3$ & $1.77)^{1.80}$ & - & - - \\
\hline & & & & & av. 1.16 \\
\hline \multirow[t]{6}{*}{$27 \mathrm{~A}$} & 5 & 8.7 & 10.73 & 9.47 & 0.82 \\
\hline & 50 & 13.1 & 8.92 & 7.66 & 1.00 \\
\hline & 245 & 26.2 & 5.75 & 4.49 & 1.17 \\
\hline & 10,212 & 642.1 & $1.25)$ & - & $\ldots$ \\
\hline & 40,011 & $2,341.0$ & $1.28)^{1.20}$ & $\cdots$ & - \\
\hline & & & & & av. 1.00 \\
\hline \multirow[t]{7}{*}{$28 \mathrm{~B}$} & .5 & 9.50 & 10.96 & 9.46 & 0.90 \\
\hline & 51 & 13.05 & 9.65 & 8.16 & 1.07 \\
\hline & 208 & 16.27 & 6.20 & 4.76 & 0.77 \\
\hline & 10,058 & 642.8 & 1.51 & -- & $\ldots$ \\
\hline & 20,069 & $1,436.0$ & $1.07)$ & & - \\
\hline & 40,092 & $2,526.0$ & $1.88)^{1.49}$ & $\cdots$ & $\ldots$ \\
\hline & & & & & av. 0.91 \\
\hline \multirow[t]{7}{*}{$30 \mathrm{~B}$} & 5 & 8.16 & 9.75 & 8.71 & 0.71 \\
\hline & 51 & 11.06 & 7.85 & 6.81 & 0.75 \\
\hline & 196 & 21.86 & 4.23 & 3.69 & 0.81 \\
\hline & 579 & 47.04 & 3.98 & 2.94 & 1.38 \\
\hline & 10,092 & 331.9 & 1.24) & $\cdots$ & - \\
\hline & 20,033 & 603.8 & $0.83)^{1.04}$ & $\ldots$ & $\cdots$ \\
\hline & & & & & av. 0.91 \\
\hline
\end{tabular}

Each entry represents the average of four animals.

Abbreviations: $\frac{100 \mathrm{~N}}{\mathrm{~L}}=\frac{100 \times \% \text { dose nuclear fraction } / \mathrm{g} \text { liver }}{\% \text { dose } / \mathrm{g} \text { liver }}$

$\left(\frac{100 \mathrm{~N}}{\mathrm{~L}}\right)_{c}=\frac{100 \mathrm{~N}}{\mathrm{~L}}$, corrected for nonspecific binding as deter-

mined by minimal values for $\frac{100 \mathrm{~N}}{\mathrm{~L}}$ found when the dose injected equaled or exceeded $10,000 \mathrm{ng} / 100 \mathrm{~g}$ body wt. When more than one "loading" dose was used, the average value for $\frac{100 \mathrm{~N}}{\mathrm{~L}}$ for the high doses indicated by brackets, was subtracted from the uncorrected $\frac{100 \mathrm{~N}}{\mathrm{~L}}$ at the low dose. Each entry represents the mean of four values. The average coefficient of variation of $\left(\frac{100 \mathrm{~N}}{\mathrm{~L}}\right)_{e}$ was $12.5 \%$. 
TABLE II

Specific Activity of Nuclei and Total Liver Homiogenate $\frac{1}{2} h$ after Injection of $\left[{ }^{225} I\right] T_{3}$

\begin{tabular}{|c|c|c|c|c|c|c|c|}
\hline \multirow[b]{2}{*}{ Rat } & \multicolumn{3}{|c|}{ Homogenate } & \multicolumn{3}{|c|}{ Nuclei } & \multirow[b]{2}{*}{$\frac{\text { Sp act Nuclei }}{\text { Sp act Homogenate }}$} \\
\hline & $\begin{array}{l}\text { Dose in } \\
\text { extract }\end{array}$ & $\begin{array}{l}T_{3} \text { in } \\
\text { extract }\end{array}$ & Sp act & $\begin{array}{l}\text { Dose in } \\
\text { extract }\end{array}$ & $\begin{array}{c}T_{3} \text { in } \\
\text { extract }\end{array}$ & Sp act & \\
\hline & $\%$ & $n g$ & $\%$ dose $/ n g T_{3}$ & $\%$ & $n g$ & $\%$ dose $/ n g T_{3}$ & \\
\hline 1 & 2.49 & 2.40 & 1.04 & 0.836 & 0.623 & 1.34 & 1.29 \\
\hline 2 & 1.91 & 2.58 & 0.740 & 0.616 & 0.687 & 0.897 & 1.21 \\
\hline 3 & 3.46 & 1.96 & 1.77 & 1.24 & 0.733 & 1.64 & 0.95 \\
\hline 4 & 2.03 & 3.54 & 0.575 & 0.616 & 1.143 & 0.539 & 0.94 \\
\hline Mean & 2.47 & 2.62 & $1.031^{*}$ & 0.827 & 0.796 & $1.117^{*}$ & 1.10 \\
\hline
\end{tabular}

Four rats were injected with $2 \mathrm{ng} / 100 \mathrm{~g}$ body weight $\left[{ }^{125} \mathrm{I}\right] \mathrm{T}_{3}$ and killed $30 \mathrm{~min}$ thereafter. Nuclei were prepared as described in Methods. The nuclear pellet was resuspended in $2.0 \mathrm{ml}$ sucrose, $(0.32 \mathrm{M})$, and the resuspended nuclei as well as a sample of the original homogenate were extracted with $95 \%$ ethanol. Four extractions with 4 vol of ethanol were sufficient to remove $95 \%$ of the counts. These ethanolic extracts were taken to dryness in a flash evaporator in vacuo at a temperature less than $45^{\circ} \mathrm{C}$. Evaporation flasks were then washed with $2 \%$ bovine serum albumin. Three $1-\mathrm{ml}$ washes were required to remove $95 \%$ of the $\left[{ }^{225} \mathrm{I}\right] \mathrm{T}_{3}$ counts. The counting rates of these solutions were then determined and portions were taken for measurement of nonradioactive $T_{3}$ by radioimmunoassay (9). * Specific activity of nuclear $T_{3}$ is not significantly different from specific activity of $T_{3}$ in total homogenate as determined by paired $t$ test.

finding indicating that the equilibrium between the cytoplasm and plasma is rapidly established.

Three possible compartmental models can be proposed to account for these relationships between $T_{3}$ in nucleus and cytoplasm (Fig. 2). Model $\mathrm{A}$ assumes that there is a two-way interchange between $T_{3}$ in cytoplasm and nuclei. Model $B$ assumes that once $T_{3}$ has been transported from the cytoplasm to the nucleus, it does not return as such to the cytoplasm but is irreversibly degraded by nuclear metabolism. Model $\mathrm{C}$ is a combination of $\mathrm{A}$ and $\mathrm{B}$ and assumes both backward movement of $T_{3}$ and irreversible metabolism of $T_{3}$ from the nuclei. It is possible to exclude Model B for the following reason. If we assume that all $\mathrm{T}_{3}$ reaching the nucleus is irreversibly metabolized, it is possible to calculate the metabolic clearance rate attributable to the postulated nuclear metabolism (Appendix A). This clearance value would be $72 \mathrm{ml} / \mathrm{hr} / 100 \mathrm{~g}$. The total metabolic clearance rate of $T_{8}$ in these animals, however, varies between 15.3 and $26.0 \mathrm{ml} / \mathrm{hr} / 100 \mathrm{~g}$ body wt, as determined in two previous studies $(10,11)$. Thus, the estimated unidirectional nuclear clearance rate is three- to five-fold greater than the metabolic clearance rate, clearly an impossible situation. The concept that $\mathrm{T}_{s}$ once delivered to the nucleus, is removed exclusively by irreversible processes (Model B) must, therefore, be rejected.

We have also performed in vitro experiments in which isotopically labeled $T_{3}$ in tracer quantities has been incubated with nuclei in the presence of ATP and cofactors at $37^{\circ} \mathrm{C}$ for $1 \mathrm{~h}$. Chromatographic analysis of the nuclei and medium showed no metabolic transformation of $T_{3}$. Thus, no support could be elicited to favor the concept that nuclei metabolize $\mathrm{T}_{8}$.

On the basis of these kinetic considerations and the negative biochemical studies, it appears reasonable to conclude that nuclear $\mathrm{T}_{\mathrm{s}}$ must exchange with cytoplasm (Models A or C). The possibility that some nuclear metabolism occurs (Model C) cannot be rigorously excluded because of the inherent limitations posed by the application of results from any in vitro model to the intact organism.

Measurement of the nuclear binding capacity of $T_{\mathrm{s}}$ (Tables I and II, Fig. 3). Theoretical considerations applied to the experiments illustrated in Fig. 1 suggest that the "equilibrium time point" at which the specific activity of nuclear $T_{3}$ is equal to the specific activity of extranuclear $T_{3}$ occurs $\frac{1}{2} \mathrm{~h}$ after the intravenous injection of tracer into normal animals with a presumed steady state level of nonradioactive $\mathrm{T}_{\mathrm{s}}{ }^{8}$ This assumption

\footnotetext{
searson, Veall, and Vetter (12) have shown that in a two-compartment system there exists a single time point when the specific activity in both compartments is the same. Thus, in the example under consideration, after the injection of tracer $T_{3}$, the specific activity in the nuclear pool is at first less than that of the cytoplasmic and plasma $T_{3}$. From a kinetic point of view, the liver cytoplasm and the plasma can be considered to be a single compartment, since there is no significant time lag in equilibration (Fig. 1 ). Subsequently, the specific activity of the nuclear $T_{3}$ will be greater than that of the extranuclear $T_{3}$. According to the principles of the analysis by Pearson et al., the single point at which the specific activity into the two kinetic pools is the same can be determined by the time point at which the rate of change of nuclear $T_{3}$ is 0 . This occurs $\frac{1}{2} \mathrm{~h}$ after injection (Fig. 1) (12).
} 


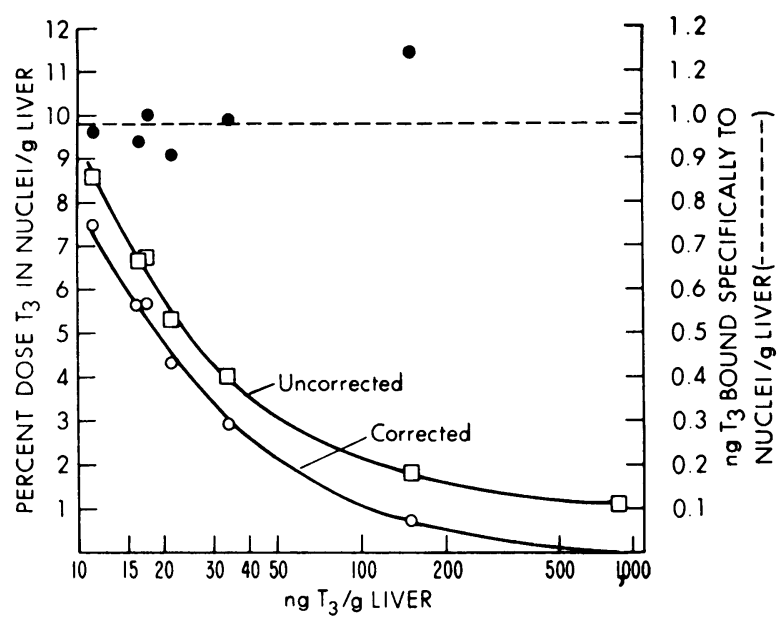

Figure 3 Calculation of nuclear binding capacity (Exp. 11). The uncorrected percent of the dose of $T_{3}$ per gram liver $(=100 \mathrm{~N} / \mathrm{L})$ and the corresponding corrected value $(100 \mathrm{~N} / \mathrm{L})_{c}$, determined by subtracting nonspecific binding, are plotted as a function of the concentration of $T_{3}$ in liver. The product of $(100 \mathrm{~N} / \mathrm{L})_{0}$ and the concentration of $T_{3}$ in liver shows that the amount of $T_{3}$ specifically bound to the nuclei is remarkably constant. It would thus appear that the limited capacity binding sites are close to saturation at endogenous concentration of $\mathrm{T}_{3}$. The first point represents the injection of trace quantities of $\mathrm{T}_{3}$.

was confirmed in experiments in which the specific activity of nuclear and cytoplasmic $T_{3}$ was measured by radioimmunoassay of $\mathrm{T}_{3}$ (Table II). Accordingly, all radioactive measurements for assessing nuclear $T_{3}$ pools were made $\frac{1}{2} \mathrm{~h}$ after injection.

As the dose of injected $\mathrm{T}_{3}$ was increased, a progressive decrease was observed in the percent of total liver radioactivity associated with the nuclear pellet (designated $100 \mathrm{~N} / \mathrm{L}$ ). These results thus confirmed our previous observations (1). For a given group of animals, the observed $100 \mathrm{~N} / \mathrm{L}$ with tracer levels of injected $\mathrm{T}_{3}$ assumed a value ranging from 7 to 13 with a mean of 9.3. As the injected dose of $T_{3}$ was increased above 10,000 $\mathrm{ng} / 100 \mathrm{~g}$ body wt, $100 \mathrm{~N}$ / $\mathrm{L}$ remained constant (see Table I). It appeared reasonable, therefore, to assume that when $100 \mathrm{~N} / \mathrm{L}$ had reached minimal values, the specificially bound material would become a negligible proportion of the total. This lower limit for $100 \mathrm{~N} / \mathrm{L}$ was therefore considered to represent largely nonspecific binding. Additional support for the concept of specific and nonspecific binding is derived from the demonstration that specifically bound nuclear $T_{3}$ is largely extracted with $0.4 \mathrm{M}$ $\mathrm{KCl}$, whereas nonspecifically bound $\mathrm{T}_{3}$ is not (13).

In order to quantitate the amount of $T_{3}$ bound to specific $\mathrm{T}_{3}$ nuclear sites, the nonradioactive concentration of $\mathrm{T}_{3}$ in the liver was determined by multiplying the liver/plasma $(\mathrm{L} / \mathrm{P})$ concentration ratio of radioactive $\mathrm{T}_{3}$ by the estimated total plasma $\mathrm{T}_{3}$ concentration. The latter value was determined from the sum of the endogenous $\mathrm{T}_{3}$ concentration, $0.84 \mathrm{ng} / \mathrm{ml}$ " and the exogenous $\mathrm{T}_{3}$ as calculated from the specific activity of the injected $\mathrm{T}_{3}$ and the counting rate of trichloroacetic acid-precipitated serum. The product of the estimated liver $\mathrm{T}_{3}$ concentration and the difference between the $100 \mathrm{~N} / \mathrm{L}$ value observed at a particular dose and the $100 \mathrm{~N} / \mathrm{L}$ value at the loading dose of $T_{3}(10,000 \mathrm{ng} / 100 \mathrm{~g}$ or greater $)$ yielded the calculated mass of $T_{3}$ specifically bound to nuclei for a particular dose of injected $\mathrm{T}_{3}$.

Results of six individual experiments are summarized in Table I. The binding capacity achieved in these experiments varied between 0.91 and $1.22 \mathrm{ng} / \mathrm{g}$ liver with a mean value of $0.99 \mathrm{ng} / \mathrm{g}$. The average concentration of DNA in the final nuclear pellet determined in 32 animals was $1.7 \mathrm{mg} / \mathrm{g}$ liver. In these animals the average recovery of DNA from the homogenate was $62 \%$. Thus the nuclear binding capacity per gram of tissue can be estimated to be approximately $1.60 \mathrm{ng} \mathrm{T}_{3}$. A graphic representation of one of these studies is presented in Fig. 3. These results indicate a sharply defined binding capacity over a wide range of injected $\mathrm{T}_{\mathrm{s}}$, from 5 to $2,000 \mathrm{ng} / 100 \mathrm{~g}$ body wt. The average pool of exchangeable $T_{3}$ in the rat can be estimated to be approximately $130 \mathrm{ng} / 100 \mathrm{~g}$ body wt. When 2,000 ng/ $100 \mathrm{~g}$ body wt are injected, the plasma and total liver concentrations are observed to increase about 15 -fold over the endogenous levels of $\mathrm{T}_{3}$. Since the endogenous concentration of $\mathrm{T}_{3}$ was not individually determined in these experiments, only a gross approximation can be offered of the percentage saturation of the nuclear sites under physiological conditions. Inspection of Table I, however, does suggest that over $70 \%$ of the sites are normally saturated.

Relationships of binding constants for $T_{3}$ and $T_{4}$. In our previous studies (1), we had demonstrated that nuclear binding sites showed a substantially greater avidity for $T_{3}$ than for $T_{4}$. To provide some quantitative estimate of the relative strength of association of $T_{3}$ and $T_{4}$, the following studies were undertaken. An additional point requiring resolution was whether the apparent cross-reactivity between $T_{s}$ and $T_{4}$ could be attributed to either in vivo conversion of $T_{4}$ to $T_{3}$ or to the existence of contaminating $T_{8}$ both in radioactive and nonradioactive preparation of $T$, used. As previously discussed, one could postulate that because of selective nuclear binding of $T_{8}$, any tracer $T_{8}$ derived from radioactive contamination of $T_{3}$ or metabolic conversion from labeled $T_{4}$ would be concentrated by the nucleus. Loading quantities of $T_{4}$ would present large amounts of nonradioactive $T_{s}$ to the nucleus and displace the tracer $T_{3}$ present.

\footnotetext{
'Based on the mean value determined in the serums of 52 rats by a radioimmunoassay technique (8). The standard error was $\pm 0.04 \mathrm{ng} / \mathrm{ml}$.
} 
In order to assess the binding characteristics of $T_{8}$, saturation experiments were analyzed according to expressions derived from the law of mass action. The ratio of $T_{s}$ bound nonspecifically to cytoplasm ( $T_{3 R}=$ total hepatocellular $T_{3}$-specific nuclear $T_{3}$ ) to specifically bound nuclear $T_{3}\left(T_{3 x}\right)$ was plotted as a function of $\mathrm{T}_{3 R}$ on the abscissa. From. Eq. 6 (Appendix B) it is apparent that the slope of the resulting function is $(1 / M)$ where $M$ is the nuclear binding capacity and intercept on the ordinate $1 / M k_{3}$, where $k_{3}$ is the equilibrium constant governing the interaction of $\mathrm{T}_{3}$ between cytoplasmic and nuclear sites as defined in Eq. 5 (Appendix $B)$. The value of $k_{s}$ can therefore be calculated. When experiment 11 was analyzed in this fashion (Fig. 4) the following results were obtained. $k_{3}=1.4 \times 10^{8} \mathrm{~kg} / \mathrm{mol}$ and $M=1.8 \mathrm{~mol} / \mathrm{kg}$.

Experiments were also carried out to assess the equilibrium constant governing the interaction of $T_{4}$ between cytoplasmic and specific nuclear sites. This was accomplished by determining the effect of increasing doses of unlabeled $T_{4}$ on the distribution of tracer $\left[{ }^{125} \mathrm{I}\right] \mathrm{T}_{\mathbf{s}}$ between cytoplasm and specific nuclear sites. From Eq. 11 (Appendix B) it is apparent that when $\left(\mathrm{T}_{3 R} / \mathrm{T}_{3 N}\right)$ is plotted as a function of $T_{4 R}$, the concentration of $T_{4}$ in the cytoplasm, the resulting function will have a limiting slope of $\left(k_{4} / k_{3}\right)$ which will extrapolate to an intercept on the ordinate with a value of $\left(1 / k_{3} M\right)$. In order to evaluate $T_{\iota R}$, tracer $\left[{ }^{181} \mathrm{I}\right] \mathrm{T}$, was injected together with

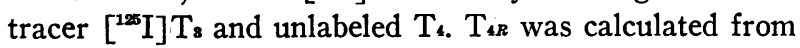
the $\mathrm{L} / \mathrm{P}$ concentration ratio of $\left[{ }^{125} \mathrm{I}\right] \mathrm{T}$, and the known

\section{TABLE III}

Displacement of Labeled $T_{4}$ by Loading Doses of Iodothyronines

\begin{tabular}{|c|c|c|}
\hline Exp & Iodothyronine injected & $\left(\frac{100 \mathrm{~N}}{\mathrm{~L}}\right)$ \\
\hline & $\mu g / 100 \mathrm{~g}$ body $w t$ & \\
\hline 31 & $\begin{array}{l}\text { A. tracer } \mathrm{T}_{4} \text { only } \\
\text { B. tracer } \mathrm{T}_{4}+80 \mu \mathrm{g} \mathrm{T}_{4} \\
\text { C. tracer } \mathrm{T}_{4}+20 \mu \mathrm{g} \mathrm{T}_{3} \\
\text { D. specifically bound }\end{array}$ & $\begin{array}{l}1.43 \\
0.91 \\
0.99 \\
0.48\end{array}$ \\
\hline 22 & $\begin{array}{l}\text { A. tracer } \mathrm{T}_{4} \text { only } \\
\text { B. tracer } \mathrm{T}_{4}+1.2 \mu \mathrm{g} \mathrm{T} \mathrm{T}_{4} \\
\text { C. tracer } \mathrm{T}_{4}+24.2 \mu \mathrm{g} \mathrm{T} \mathrm{T}_{4} \\
\text { D. tracer } \mathrm{T}_{4}+20 \mu \mathrm{g} \mathrm{\textrm {T } _ { 3 }} \\
\text { E. specifically bound } \mathrm{T}_{3}\end{array}$ & $\begin{array}{l}1.19 \\
0.82 \\
0.73 \\
0.74 \\
0.48\end{array}$ \\
\hline
\end{tabular}

For definition of $\frac{100 \mathrm{~N}}{\mathrm{~L}}$, see legend to Table I.

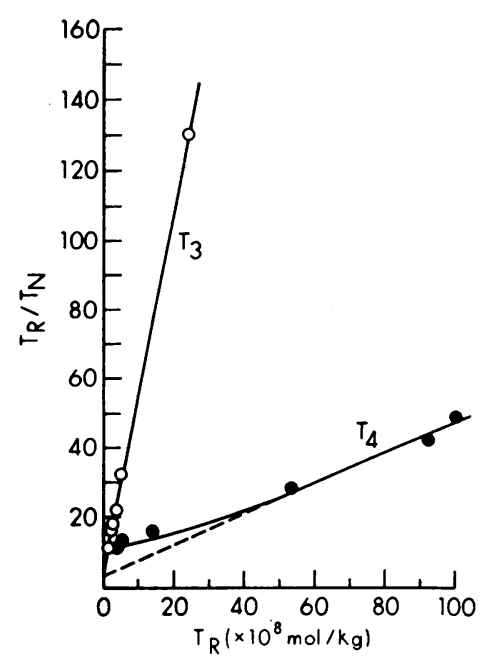

FIGURE $4 T_{R}$, the molar concentration of $T_{3}$ or $T_{4}$ in the cytoplasm, is plotted against the ratio of $\mathrm{T}_{R}$ to $\mathrm{T}_{N}$, the molar concentration of iodothyronine specifically bound in the nuclei. In the case of $T_{3}$, there is a linear relationship, consonant with theoretical expectation for a single set of nuclear binding sites. In the case of $T_{4}$, the relationship is not linear at the lower doses of $T_{4}$ injected, since the predominant effect on partition between nucleus and cytoplasm is determined by the endogenous levels of $T_{3}$ in the cell. Euthyroid animals were used in these studies and each point represents the mean of four animals. See the text for details of design, calculations, and interpretation of results.

plasma concentration of $T_{4}$. The results are illustrated in Fig. 4 in an experiment with the same group of animals used in estimating $k_{3}$. It was estimated that $k_{4}=1.1 \times 10^{7} \mathrm{~mol} / \mathrm{kg}$ and that the ratio $k_{3}$ to $k_{4}$ was 13 . In this analysis the assumption is made that the equilibrium time point for $T_{4}$ is the same as for $T_{3}$. Serial measurements of nuclear $\left[{ }^{121} \mathrm{I}\right] \mathrm{T}$, have verified this assumption, with maximal radioactivity occurring between 15 and $30 \mathrm{~min}$ after intravenous injection of tracer.

The following experiments were carried out to evaluate the possibility that the observed cross-reactivity between $T_{4}$ and $T_{3}$ was due exclusively to contamination of the $T_{4}$ preparation with $T_{3}$ or the conversion of $T_{4}$ to $T_{3}$ in vivo. In Table III we have summarized two experiments in which tracer amounts of radioactive $T$. were injected alone as well as with loading doses of $T_{4}$ and $\mathrm{T}_{\mathbf{3}}$. With loading doses of either $\mathrm{T}_{4}$ or $\mathrm{T}_{\mathbf{3}}$, approximately $33 \%$ and $40 \%$ of the nuclear radioactivity at tracer levels was displaced in Exp. 31 and 22, respectively. Chromatographic analysis of radioactivity of nuclear extracts from animals injected with tracer $\mathrm{T}_{4}$ only (Exp. 31, group A), however, showed that only $7 \%$ of nuclear radioactivity was in the form of $\mathrm{T}_{3}$. The remainder was $T_{4}$. Thus, on the basis of $T_{3}$ contamination or conversion, one could expect only a $7 \%$ decrease in 
the counting rate of nuclear radioactivity with loading doses of $T_{4}$. Therefore, intrinsic cross-reactivity by nuclear sites for $T_{8}$ and $T_{4}$ exists and only a fraction of the observed cross-reactivity can be attributed to $T_{4}$ contamination or conversion to $\mathrm{T}_{3}$.

The data in Table III can also be used to provide an independent estimate of the ratio of $k_{3}$ to $k_{4}$. Thus, from the law of mass action, it can be shown that:

$$
\left(\frac{k_{3}}{k_{4}}\right)=\frac{\left(\mathrm{T}_{3 N} / \mathrm{T}_{3 R}\right)}{\left(\mathrm{T}_{4 N} / \mathrm{T}_{4 R}\right)} \text {. }
$$

If we assume from Table I that the average (corrected) percent bound to the nucleus is 8.61 , then it follows that $\left(\mathrm{T}_{3 x} / \mathrm{T}_{8 R}\right)$ is equal to $8.61 / 91.39=0.0942$. From Table III, similar calculations of specific binding would indicate that the corresponding ratio for $T_{4}$ is 0.00472 . Hence, $k_{s} / k_{4}$ is equal to 20 . This contrasts with the value of 13 calculated from the experiments illustrated in Fig. 4. Whereas the agreement between the two values is not ideal, the estimates have the same order of magnitude and are useful approximations of the relative avidity of the nuclear sites for $T_{3}$ and $T_{4}$ in cytoplasm.

It should be emphasized that the values for $k_{3}$ and $k_{4}$ provided in these studies are not association constants since the equilibrium assessed was between the nuclei and cytoplasm, not between the nuclei and an aqueous medium. Nevertheless, if one knows the strength of protein binding as determined by equilibrium dialysis studies for $T_{3}$ and $T_{4}$, one can calculate the order of magnitude of the apparent association constants: The approximate dialyzable fraction for $T_{3}\left(D_{3}\right)$ is 0.003 and that for $T_{4}$ $\left(\mathrm{DF}_{4}\right)$ is $0.0003(11)$. If we designate $k^{\prime}{ }_{3 N}$ as the apparent association constant

$$
\frac{k^{\prime}{ }_{3 N}}{k_{3 N}}=\frac{(L / P)}{\left(D F_{3}\right)}=\frac{10}{0.003} .
$$

if $k_{3 \mathrm{~N}}=1.4 \times 10^{8}, k_{\mathrm{sN}}^{\prime}=(10 / 0.003) \times\left(1.4 \times 10^{8}\right)=$ $4.7 \times 10^{11}$ liters $/ \mathrm{mol}$. Similarly, since $D F_{4}=0.0003$ and the average $(L / P)_{4}=0.6, \quad k^{\prime}{ }_{4 N}=0.6 / 0.003 \times 1.1 \times 10^{7}$ $=2.2 \times 10^{10}$. The ratio of $k^{\prime} s$ to $k^{\prime} 4$ is therefore about 21 . Alternatively, if one accepts the ratio of $\left(k_{8} / k_{4}\right)=20$ as derived from Eq. 12, then similar considerations can be applied to show that $\left(k^{\prime}{ }^{\prime} / k_{4}^{\prime}\right)=33.3$

\section{DISCUSSION}

The demonstration of specific nuclear binding sites for $T_{s}$ derives potential significance, not only from previously cited data by Tata and associates indicating early nuclear events after pulse injection of $T_{3}$, but also from rapidly accumulating evidence, that a number of steroid hormones exert their action at a nuclear level $(14,15)$. The hypothesis has been advanced by several investigators that progesterone, estradiol, dihydrotestosterone, and cortisol are first bound to a cytosol receptor and that the cytosol receptor hormone complex in turn is transferred to the nucleus. There remains considerable controversy as to whether the steroid receptor in the nuclear chromatin fraction is the same as that in the cytosol.

These considerations raise the problem as to whether specific cytosol receptors also exist for $T_{s}$. This problem is currently under intensive study in our laboratory. A number of investigators have reported $\mathrm{T}_{3}$ and $\mathrm{T}_{4}$ cytosol-binding proteins (16-19) but the role of these proteins has not been well defined. In previous studies with tracer and loading doses of $T_{3}$, we have analyzed the distribution of $T_{3}$ in cytosol and other subcellular fractions. No evidence was found for appreciable quantities of specific binding sites other than those in nuclei. It is possible, however, that the concentration of cytosol binding sites was too small to allow detection. Regardless of whether $T_{3}$ in the cytoplasm is bound specifically or nonspecifically, the kinetic data presented in the current report indicate a relatively rapid two-way shuttle of $T_{s}$ between the nucleus and the cytoplasm. One-way transport of $T_{8}$ from cytosol to nucleus is not possible, because a model with one-way transport leads to an estimate of the hypothetical unidirectional clearance rate by nuclei several times greater than the total metabolic clearance rate of the entire rat. Moreover, no evidence was found in experiments in vitro to support the concept that nuclei can metabolize $\mathrm{T}_{\mathrm{s}}$.

Our data indicate that only $9-12 \%$ of cellular radioactivity is recovered with the nuclear pellet. On the basis of determinations of DNA in the tissue homogenate and in the nuclear pellet, we estimate that approximately $38 \%$ of the nuclear fraction is lost in the purification process. Thus, our data suggest that from $14-19 \%$ of the total radioactivity in the cell is associated with the nucleus. These findings explain the relative stability of the total $\mathrm{L} / \mathrm{P}$ concentration ratio with increasing doses of injected $\mathrm{T}_{3}$. Since no more than $19 \%$ of total cellular binding is due to specific nuclear binding sites, one would anticipate that maximal doses of $\mathrm{T}_{3}$ would reduce the total $\mathrm{L} / \mathrm{P}$ ratio to $81 \%$ of the baseline value. Inherent biological variation makes it difficult to detect changes in this range unless large numbers of animals are used.

Siegel and Tobias (20) have reported preferential localization of $\left[{ }^{125} \mathrm{I}\right] \mathrm{T}_{4}$ to the nuclei of kidney tumor cells grown in tissue culture. No studies were carried out with $\left[{ }^{125} \mathrm{I}\right] \mathrm{T}_{3}$. Saturation studies were not performed, and no information was provided about possible losses of radioactivity from the cells during fixation and radioautography. The concentration of $T_{t}$ in the culture medium was at least 50 times the plasma concentration of $T_{4}$ in mammalian serum. Griswold, Fischer, and Cohen (21) have recently reported temperature-dependent intracellular distribution of $T_{4}$ in amphibian liver. After in- 
jection of $\mathrm{T}_{4}$ at $25^{\circ} \mathrm{C}$, from $40-50 \%$ of tadpole liver $\mathrm{T}_{4}$ was associated with nuclei. The molar concentration of $\mathrm{T}$. bound to nuclei was approximately 10,000 times greater than the molar concentration of iodothyronine bound to specific nuclear sites in the rat. Again, no saturation studies were carried out. It is therefore difficult to compare the findings in these reports to the results of our studies.

If we estimate that each gram of liver contains $3.34 \times$ $10^{8}$ nuclei (22) and if we assume that there has been a $38 \%$ loss of nuclear material during preparation, we can calculate from the nuclear binding capacity $(0.99 \mathrm{ng}$ $\mathrm{T}_{3} / \mathrm{g}$ liver) that each nucleus contains approximately 4,400 molecules of $T_{3}$. If we further assume that each nucleus contains 254-cm double-stranded DNA (23) we can also estimate that on the average there is one molecule of $T_{3}$ for every $575 \mu \mathrm{m}$ of DNA. It will be of interest to determine whether similar quantitative relationships exist between $T_{3}$ and DNA in other tissues.

The results of the present set of experiments indicate that the nuclear sites are not absolutely specific for $\mathrm{T}_{\mathbf{8}}$ since they also interact with $T_{4}$. We had previously queried whether the slight depression in the nuclear binding of labeled tracer $T_{4}$ by the injection of large doses of nonradioactive $T_{4}$ might not have been caused by contaminating $T_{s}$ in the $T_{4}$ preparations used and the conversion of $T_{4}$ to $T_{3}$ in vivo (1). The chromatographic studies summarized in Table III effectively exclude this possibility. On the other hand, the current data suggest that the affinity of nuclear sites for $T_{s}$ is at least 20 times greater than their affinity for $T_{4}$. The relative specificity of nuclear sites for $T_{3}$ supports recent metabolic studies which have suggested that the hormonal action of $T_{4}$ is derived largely, if not exclusively, from its conversion to $T_{3}(4,10,24)$. The concept that $\mathrm{T}_{3}$ is the "active" hormone was first proposed by Gross and Pitt-Rivers (25) but subsequently rejected on the basis of a report by Lassiter and Stanbury (26) negating $T_{4}$ to $T_{3}$ conversion in man. The concept of $T_{4}$ to $T_{3}$ conversion was subsequently revived by Braverman, Ingbar and Sterling (28) who found significant conversion of $T_{4}$ to $T_{3}$ in athyreotic subjects maintained on $\mathrm{T}_{\mathrm{s}}$.

If one assumes that metabolic effects of $\mathrm{T}_{4}$ and $\mathrm{T}_{3}$ are equivalent, molecule-for-molecule bound to the specific nuclear sites, it is possible to provide an estimate of the overall intrinsic metabolic contributions of $T_{3}$ and $T_{4}$ (independent of its conversion to $T_{3}$ ) both in rat and in man. On the basis of the isotopic measurements and the known concentration of $T_{3}$ and $T_{4}$ in plasma, it is possible to calculate that the quantity of $T_{3}$ bound specifically to nuclei per gram of rat liver is $20.3 \times 10^{-13} \mathrm{~mol}$, whereas the corresponding mass of $T_{4}$ specifically bound is $2.83 \times 10^{-18} \mathrm{~mol}$. Thus one would estimate that the contribution of $T_{3}$ to hormonal effects of $T_{3}$ exceeds that of $T_{4}$ by a factor of 7.1. It is possible to provide a similar estimate in man, since in man the ratio of $T_{4} / T_{8}$ plasma binding $(=10)$ and the approximate molar ratio of total $\mathrm{T}_{4} / \mathrm{T}_{3}(=45)$ are known, and the ratio of the association constants of $T_{3}$ to $T_{4}$ is probably in the same range as in the rat $(=21-33)$. Calculations based on these data suggest that in normal man the contribution to hormonal effect derived from the total blood production of $T_{3}$ exceeds the intrinsic contribution of $T_{4}$ by a factor in the range of 4.7-7.3. In other words, from 12 to $18 \%$ of the total metabolic effect of the iodothyronines may be due to $\mathrm{T}_{4}$. Thus, a change in one $\mu \mathrm{g} / 100 \mathrm{ml} \mathrm{T}$. would be approximately equivalent to $2.6-3.7 \mathrm{ng} \mathrm{T}_{3} / 100$ ml.

The demonstration of limited-capacity high-affinity $\mathrm{T}_{\mathbf{s}}$ binding sites per se does not prove the biological relevance of such sites. Nevertheless, on the basis of Tata's demonstration of early nuclear response to $T_{3}$, the role of nuclei in mediating the effects of other hormones, and the existence of nuclear $T_{3}$ sites in a wide variety of tissues, it appears highly likely that these sites are responsible for initiating biochemical reactions that result in the expression of hormonal activity. If so, our data raise an interesting set of questions about the quantitative relationship between the concentration of the $T_{3-}$ nuclear complex and the hormonal effect. Our data suggest that at least $70 \%$ of the nuclear sites are occupied at endogenous concentrations of plasma $\mathrm{T}_{8}$. For hyperthyroidism one one or more of the following would have to occur: (a) that the occupancy of the residual sites exerts a proportionately greater quantitative effect, $(b)$ that there are qualitative or quantitative differences in nuclear $T_{3}$ binding in hyperthyroidism, (c) that the transition from the hypothyroid to the euthyroid state is mechanically different from the transition from the euthyroid to the hyperthyroid state. Further experiments are required to resolve these issues.

Lastly, it is important to emphasize the limitations in our analysis. The numercial values for the binding capacity and association constants are approximations only, since their determination is limited by kinetic assumptions, mean group values for plasma hormone concentrations rather than individual animal assays, and the experimental error inherent in the techniques used. Thus, in this study, we did not correct for DNA losses in the preparation of individual nuclear pellets, but simply assumed a mean loss. Also, the possibility that some $T_{s}$ dissociates immediately from the nucleus as soon as it is placed in hypertonic sucrose cannot be excluded nor easily tested by available techniques. Moreover, it is important to emphasize that the concept of nuclear binding as used in this study is operationally defined as the mechanism for the selective concentration of $T_{s}$ and related compounds at special nuclear sites. Thus, while an analogy is implicit between this process and more con- 
ventional examples of binding, such as the binding of hormones by plasma proteins, care should be exercised not to endow kinetic characteristics with specific mechanistic interpretations. For example, we have not excluded the possibility that either the association or dissociation of $\mathrm{T}_{3}$ with the sites requires the expenditure of metabolic energy.

\section{APPENDIX A}

It follows from the definitions of the model system B (Fig. 1) that

$$
N_{\iota}=k_{1} \int_{0}^{t} R d t-k_{2} \int_{0}^{t} N d t
$$

where $N_{t}$ equals the percent of the dose injected in the nuclei per gram tissue at the time $t$ in hours after the injection; $R$, the percent of the injected dose per gram tissue in the cytoplasm, $k_{1}$ the fractional rate $\left(\mathrm{h}^{-1}\right)$ of transfer of $\mathrm{T}_{3}$ from compartment $R$ to $N$; and $k_{2}$, the fractional irreversible rate of disposition of $T_{3}$ from the nuclei $\left(h^{-1}\right)$. Eq. 1 simply states that the nuclear content of $T_{3}$ at any time $t$ represents the difference between the total amount of radioactivity brought to the nuclei and the total amount of $T_{3}$ removed from the nuclei.

Similarly, it follows that the difference in nuclear counts between two time intervals after the injection, $t$ and $t^{\prime}$, is given by the expression

$$
N_{t}-N_{t^{\prime}}=k_{1} \int_{t^{\prime}}^{t} R d t-k_{2} \int_{t^{\prime}}^{t} N d t .
$$

The rate constants $k_{1}$ and $k_{2}$ can be evaluated by solution of simultaneous equations 1 and 2. Thus, let $t^{\prime}$ equal $0.5 \mathrm{~h}$ and $t, 4.0 \mathrm{~h}$. The integral expressions in Eqs. 1 and 2 can be evaluated graphically. The value of both $N_{0.5}$ and $N_{4}$ are known. Solution of the equations will indicate that $k_{1}$ equals $0.94 / \mathrm{h}$ and $k_{2}$ equals $8.53 / \mathrm{h}$. The specific activity of $T_{3}$ is uniform throughout the system under consideration at approximately $30 \mathrm{~min}$, the time at which nuclear radioactivity is maximal, i.e., at the time that the rate of delivery of radioactivity to the nucleus equals the rate of removal from the nucleus. From this information we can calculate the unidirectional clearance $\left({ }_{N} \mathrm{Cl}_{\mathrm{T}_{3}}\right)$ of $\mathrm{T}_{3}$ from the nuclei. Thus,

$$
{ }_{N} \mathrm{Cl}_{T_{3}}=\rho(N / P) k_{2},
$$

where $(N / P)=$ ratio of nuclei $\mathrm{T}_{3}$ per gram liver to the concentration of $T_{3}$ per milliliter of plasma and $\rho=$ liver weight in grams per $100 \mathrm{~g}$ of body weight. On the basis of previous studies with similar rats in this laboratory (10), $\rho$ has been estimated to be $4.36 . N / P$ at $30 \mathrm{~min}$ is 1.93 and $k_{2} 8.53 / \mathrm{h}$. Thus ${ }_{x} \mathrm{Cl}_{\mathrm{T}_{3}}=71.8 \mathrm{ml} / \mathrm{h} / 100 \mathrm{~g}$ body wt.

\section{APPENDIX B}

In analyzing the interactions of increasing concentrations of $T_{3}$ with nuclear sites, the contributions of endogenous $T_{4}$ can be disregarded as a first approximation. Thus,

$$
\mathrm{T}_{3 R}+N \rightleftharpoons \mathrm{T}_{3 N}
$$

where $\mathrm{T}_{3 R}$ is the concentration of $\mathrm{T}_{3}$ in cytoplasm; $\mathrm{T}_{8 N}$, the concentration of $T_{s}$ in nuclei; and $N$, the concentration of unoccupied nuclear binding sites. From the law of mass action one can define an equilibrium constant $k_{3}$ such that

$$
k_{3}=\frac{\mathrm{T}_{3 N}}{(N)\left(\mathrm{T}_{3 R}\right)} \text {. }
$$

Since $N=M-\mathrm{T}_{3 N}$, where $M$ is the nuclear binding capacity, one can substitute into Eq. 5 and rearrange terms so that

$$
\left(\frac{\mathrm{T}_{3 R}}{\mathrm{~T}_{3 N}}\right)=\mathrm{T}_{3 R}\left(\frac{1}{M}\right)+\frac{1}{k_{3} M} .
$$

From the slope and ordinate intercept of a plot of $\left(\mathrm{T}_{3 R} / \mathrm{T}_{3 N}\right)$ as a function of $\mathrm{T}_{3 R}, M$, and $k_{3}$ can be determined.

In a system in which the contributing $T_{4}$ cannot be ignored, i.e. one in which progressively increasing doses of $T_{4}$ are injected, the relationship between the tracer distributing $\left.{ }^{125} \mathrm{I}\right] \mathrm{T}_{3}$ in cytoplasm and nucleus $\mathrm{T}_{3 R} / \mathrm{T}_{3 N}$ can be related to the concentration of $\mathrm{T}_{4}$ in cytoplasm $\left(\mathrm{T}_{4 R}\right)$ as follows. Since both $T_{3}$ and $T_{4}$ occupy the same sites it is apparent that

$$
N=\frac{\mathrm{T}_{3 N}}{\left(\mathrm{~T}_{3 R}\right) k_{3}}=\frac{\mathrm{T}_{4 N}}{\left(\mathrm{~T}_{4 R}\right)\left(k_{4}\right)},
$$

where the subscript 4 refers to $T_{4}$, in a notation analogous to) that for $T_{3}$.

From Eq. 7 it follows that

$$
k_{4}=\frac{\mathrm{T}_{4 N}}{\mathrm{~T}_{4 R}\left(M-\mathrm{T}_{4 N}-\mathrm{T}_{3 N}\right)} .
$$

Rearrangement of Eq. 8 leads to

$$
\left(\frac{\mathrm{T}_{4 R}}{\mathrm{~T}_{4 N}}\right)=\frac{\left(\frac{1}{k_{4}}\right)+\mathrm{T}_{4 R}}{M-\mathrm{T}_{3 N}} .
$$

From Eq. 7 it is apparent that

$$
\left(\frac{\mathrm{T}_{4 R}}{\mathrm{~T}_{4 N}}\right)=\frac{\mathrm{T}_{3 R}}{\mathrm{~T}_{3 N}} \cdot \frac{k_{3}}{k_{4}} .
$$

Substituting into Eq. 9,

$$
\left(\frac{\mathrm{T}_{3 R}}{\mathrm{~T}_{3 N}}\right)=\frac{\frac{1}{k_{3}}+\frac{k_{4}}{k_{3}}\left(T_{4 R}\right)}{M-\mathrm{T}_{3 N}} .
$$

A.s the mass of injected $\mathrm{T}_{4}$ is progressively increased $\mathrm{T}_{3 N}$ approaches 0 and

$$
\left(\frac{\mathrm{T}_{3 R}}{\mathrm{~T}_{3 N}}\right) \approx \frac{1}{k_{3} M}+\frac{\left(k_{4} / k_{3}\right)\left(\mathrm{T}_{4 R}\right)}{M} .
$$

\section{ACKNOWLEDGMENTS}

The authors thank Mr. Jose Guerra and Mr. Francisco Martinez for their expert technical assistance and Mrs. Mary Ann Mullen for her secretarial support. Mr. Barry Shapiro illustrated the figures.

This work was supported in part by National Institutes of Health grant AM 15421-13 and Department of the Army contract DA-49-193-MD-2967.

\section{REFERENCES}

1. Oppenheimer, J. H., D. Koerner, H. L. Schwartz, and M. I. Surks. 1972. Specific nuclear triiodothyronine 
binding sites in rat liver and kidney. J. Clin. Endocrinol. Metab. $35: 330$.

2. Tata, J. R., and C. C. Widnell. 1966. Ribonucleic acid synthesis during the early action of thyroid hormones. Biochem. J. 98: 604 .

3. Widnell, C. C., and J. R. Tata. 1966. A procedure for the isolation of enzymatically active rat-liver nuclei. Biochem. J. $92: 313$.

4. Schwartz, H. L., M. I. Surks, and J. H. Oppenheimer. 1971. Quantitation of extrathyroidal conversion of Lthyroxine of $3,5,3^{\prime}$-triiodo-L-thyronine in the rat. $J$. Clin. Invest. 50: 1124

5. Volpert, E. M., M. Martinez, and J. H. Oppenheimer. 1967. Radioiodinated impurities in commercial preparations of ${ }^{181}$ I-thyroxine and their effect on the measurement of free thyroxine in human serum by equilibrium dialysis. J. Clin. Endocrinol. Metab. 27: 421.

6. Bellabarba, D., R. E. Peterson, and K. Sterling. 1968. An improved method for chromatography of iodothyronines. J. Clin. Endocrinol. Metab. 28: 305.

7. Burton, K. 1956. A study of the conditions and mechanism of the diphenylamine reaction for the colorimetric estimation of dioxyribonucleic acid. Biochem. J. 62: 315.

8. Surks, M. I., A. R. Schadlow, and J. H. Oppenheimer. 1972. A new radioimmunoassay for plasma L-triiodothyronine: Measurement in thyroid disease and in patients maintained on hormonal replacement. J. Clin. Invest. $51: 3104$.

9. Koerner, D., H. L. Schwartz, M. I. Surks, and J. H. Oppenheimer. 1972. Nuclear binding of $T_{3}$ : comparison between in vivo and in vitro interactions. Program of the 48th Meeting of the American Thyroid Association (Chicago), p. 25 (Abstr.).

10. Oppenheimer, J. H., H. L. Schwartz, and M. I. Surks. 1972. Propylthiouracil inhibits the conversion of Lthyroxine to L-triiodothyronine. An explanation of the antithyroxine effect of propylthiouracil and evidence supporting the concept that triiodothyronine is the active thyroid hormone. J. Clin. Invest. 51: 2493.

11. Oppenheimer, J. H., H. L. Schwartz, H. C. Shapiro, G. Bernstein, and M. I Surks. 1970. Differences in primary cellular factors influencing the metabolism and distribution of 3,5,3'-L-triiodothyronine and L-thyroxine. J. Clin. Invest. $49: 1016$.

12. Pearson, J. D., N .Veall, and H. Vetter. 1958. A practical method for plasma albumin turnover studies. Strahlentherapie. 38: 290.

13. Surks, M. I., D. Koerner, W. Dillman, and J. H. Oppenheimer. 1973. Limited capacity binding sites for L-triiodothyronine $\left(T_{3}\right)$ in rat liver nuclei: Localization to the chromatin and partial characterization of the $T_{\mathbf{8}}$ chromatin complex. J. Biol. Chem. 248: 7066.
14. Feldman, D., J. W. Funder, and I. S. Edelman. 1972. Subcellular mechanisms in the action of adrenal steroids. Am. J. Med. 53: 545.

15. Raspe, G., ed. 1970. The Schering workshop on steroid hormone "receptors." In Advances in Biosciences. Pergamon Press Ltd., Oxford.

16. Hamada, S., K. Torizuka, and T. Miyake. 1966. Cellular thyroxine binding proteins and their physiological significance. Gunma Symp. Endocrinol. 3: 153.

17. Hamada, S., and S. H. Ingbar. 1971. Cellular binding proteins for thyroxine $\left(T_{4}\right)$ and triiodothyronine $\left(T_{3}\right)$ in rat liver cytosol. Program of the 53rd Meeting of the Endocrine Society (San Francisco) (Abstr. 134).

18. Handwerger, B. S., and P. J. Davis. 1972. Properties of cytosol proteins which bind thyroxine $\left(T_{4}\right)$ and triiodothyronine $\left(\mathrm{T}_{3}\right)$. Clin. Res. 20: 429 (Abstr.).

19. Sterling, K., M. A. Brenner, V. F. Saldanha, and P. O. Milch. 1972. Cytosol binding protein (CBP) of thyroxine in human and rat kidney tissues. Program of the 48th Meeting of the American Thyroid Association (Chicago). 43 (Abstr.).

20. Siegel, E., and C. A. Tobias. 1966. End-organ effects of thyroid hormones: subcellular interactions in cultured cells. Science (Wash., D. C.). 153: 763.

21. Griswold, M. D., M. S. Fischer, and P. P. Cohen. 1972. Temperature-dependent intracellular distribution of thyroxine in amphibian liver. Proc. Soc. Natl. Acad. Sci. U.S. A. $69: 1486$.

22. Leuchtenberger, C., R. Vendrely, and C. Vendrely. 1951. A comparison of the content of desoxyribosenucleic acid (DNA) in isolated animal nuclei by cytochemical and chemical methods. Proc. Natl. Acad. Sci. U. S. A. $37: 33$.

23. DuPraw, E. J. 1968. Cell and Molecular Biology. Academic Press, Inc., New York. 521.

24. Surks, M. I., A. R. Schadlow, J. M. Stock, and J. H. Oppenheimer. 1973. Determination of iodothyronine absorption and conversion of L-thyroxine $\left(T_{4}\right)$ to $\mathrm{L}$-triiodothyronine $\left(\mathrm{T}_{3}\right)$ using turnover rate techniques. $J$. Clin. Invest. $52: 805$.

25. Gross, J., and R. Pitt-Rivers. 1952. The identification of $3: 5: 3^{\prime}$-L-triiodothyronine in human plasma. Lancet. $1: 439$.

26. Lassiter, W. C., and J. B. Stanbury. 1958. The in vivo conversion of thyroxine to $3: 5: 3^{\prime}$-triiodothyronine. $J$. Clin. Endocrinol. Metab. 18 : 903.

27. Braverman, L. E., S. H. Ingbar, and K. Sterling. 1970. Conversion of thyroxine $\left(\mathrm{T}_{4}\right)$ to triiodothyronine $\left(\mathrm{T}_{3}\right)$ in athyretotic human subjects. J. Clin. Invest. 49: 855.

28. Bernstein, G., S. A. Artz, J. Hasen, and J. H. Oppenheimer. 1968. Hepatic accumulation of ${ }^{125} \mathrm{I}$-thyroxine in the rat: augmentation by phenobarbital and chlordane. Endocrinology. 82: 406. 